\title{
AN EXPLORATION OF FACTORS INFLUENCING AMBUlanCE AND EMERGENCY NuRSES' Protocol AdHERENCE IN THE NetherLands
}

\author{
Authors: Remco H.A. Ebben, MSc, RN, Lilian C.M. Vloet, PhD, RN, Donna M.J. Schalk, MSc, Joke A.J. Mintjes-de \\ Groot, PhD, RN, and Theo van Achterberg, PhD, RN, FEANS, Nijmegen, the Netherlands
}

Introduction: Adherence to ambulance and ED protocols is often suboptimal. Insight into factors influencing adherence is a requisite for improvement of adherence. This study aims to gain an in-depth understanding of factors that influence ambulance and emergency nurses' adherence to protocols.

Methods: Semi-structured interviews were held with ambulance nurses, emergency nurses, and physicians $(N=20)$ with medical end responsibility in the Netherlands to explore influencing factors. Content analysis was used to identify influencing factors.

Results: The main influencing factors for adherence were individual factors, including individual (clinical) experience, awareness, and the preference of following local protocols instead of national protocols. Organizational or external factors were involvement in protocol development, training and education, control mechanisms for adherence, and physicians' interest. Also of influence were protocol characteristics including integration of the advanced trauma life support approach, being in accordance with daily practice, and the generality of the content. Influencing factors could be a barrier as well as a facilitator for adherence.

Discussion: Factors influencing ambulance and emergency nurses' protocol adherence could be assigned to individual, organizational, and external categories, as well as to protocol characteristics. To improve adherence, implementation strategies should be tailored to identified factors. Multifaceted implementation strategies will be needed to improve adherence.

Key words: Emergency nursing; Emergency medical services; Guideline adherence
Remco H. A. Ebben, Research Group for Acute Care, Faculty of Health and Social Studies, HAN University of Applied Sciences, Nijmegen, the Netherlands. Lilian C. M. Vloet, Research Group for Acute Care, Faculty of Health and Social Studies, HAN University of Applied Sciences, Nijmegen, the Netherlands, and Canisius Wilhelmina Hospital, Nijmegen, the Netherlands.

Donna M. J. Schalk, Unit Internal Audit, Institute of Quality Assurance and Patient Safety, Radboud University Nijmegen Medical Centre, Nijmegen, the Netherlands.

Joke A. J. Mintjes-de Groot, Research Group for Acute Care, Faculty of Health and Social Studies, HAN University of Applied Sciences, Nijmegen, the Netherlands. Theo van Achterberg, Scientific Institute for Quality of Healthcare, Radboud University Nijmegen Medical Centre, Nijmegen, the Netherlands.

The study was funded by a grant from the Netherlands Organisation for Health Research and Development ZonMw (project No. 8271.2001). ZonMw had no involvement in the study design, interpretation of results, and writing of the manuscript.

For correspondence, write: Remco H. A. Ebben, MSc, RN, Research Group for Acute Care, Faculty of Health and Social Studies, HAN University of Applied Sciences, PO Box 6960, 6503 GL, Nijmegen, The Netherlands; E-mail: remco.ebben@han.nl.

J Emerg Nurs 2014;40:124-30.

Available online 6 November 2012.

0099-1767/\$36.00

Copyright $@ 2014$ Emergency Nurses Association. Published by Elsevier Inc. All rights reserved.

http://dx.doi.org/10.1016/j.jen.2012.09.008
7 uidelines and protocols are developed and implemented to improve quality of care and to reduce variation of practice. $^{1}$ A guideline consists of systematically developed statements for decision making that are based on research results, clinical experience, patient preferences, and available resources. ${ }^{2}$ A protocol is a specification of a guideline that exactly formulates how to act and which steps to follow. ${ }^{2}$ Despite the existence of guidelines and protocols, a gap between recommended care and delivered care often exists, ${ }^{1}$ which may suggest suboptimal adherence to these guidelines and protocols. For the ambulance and ED settings, adherence to guidelines and protocols regarding cardiopulmonary resuscitation, cervical spine immobilization, initial electrocardiographic screening, pain management, hygiene precautions, and domestic violence screening shows a wide variation. ${ }^{3-9}$ It is important to gain in-depth understanding of reasons for non-adherence to guidelines and protocols to reduce the incidence of errors occurring through omission of treatment, as well as to ensure that a maximal number of patients receive the benefit of appropriate treatment. ${ }^{10}$ Furthermore, implementation strategies to improve adherence can be tailored to these influencing factors. From an implementation perspective, influencing factors can be categorized into 


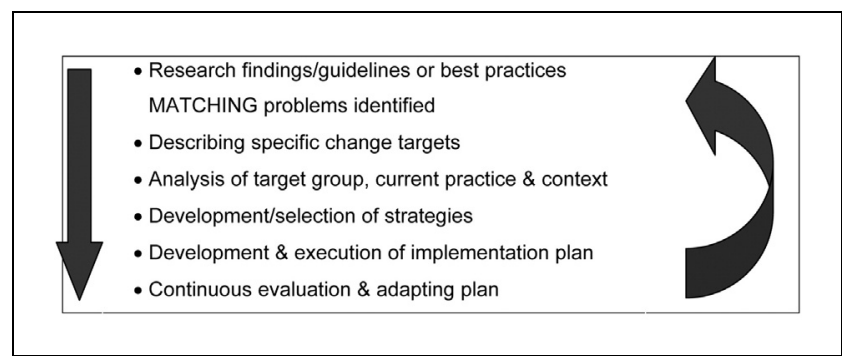

FIGURE 1

Grol's model for effective implementation. ${ }^{1}$

the individual professional, the organization, the external context (social/physical/regulations/policies), and the innovation (eg, guidelines and protocols). ${ }^{2}$

Previous studies in the prehospital and ED settings associated patient-related factors (age, gender, weight, presentation of disease, comorbidity), professional-related factors (knowledge, attitude, communication), organizational factors (location and type of emergency department [urban or rural], presence of a physician), and protocolrelated factors (lacking or inadequate protocols) with adherence to guidelines and protocols. 5,9,11-18

Despite the existence of these studies, in-depth understanding of factors that influence nurses' adherence to ambulance and ED protocols is lacking. Therefore the study objective was to gain an in-depth understanding of factors that influence ambulance nurses' and emergency nurses' adherence to ambulance and ED protocols.

\section{Methods}

\section{SETTING}

In the Netherlands, prehospital ambulance care is provided 24 hours per day by 25 emergency medical services (EMSs). Geographical dispersion of these EMSs is based on the starting point that $95 \%$ of the urgent patients can be reached within 15 minutes after the first call. Regular ambulances are staffed with 1 driver and 1 registered ambulance nurse. Registered nurses become qualified as an ambulance nurse after a specific national training course. Ambulance nurses work autonomously and are allowed to administer medical treatment based on their national protocol, without direct consultation of an EMS physician. Though not directly involved on site, the EMS physician has medical end responsibility for the provided care. Dutch emergency departments provide emergency care 24 hours per day for all types of patients. At the time of the study, there were 103 emergency departments in the Netherlands, which are staffed with emergency nurses, emergency physicians, and medical residents. Registered nurses follow additional emergency training to become qualified as an emergency nurse. Medical end responsibility varies per emergency department, although the medical end responsibility is shifting toward the emergency physician.

To support clinical practice by ambulance nurses, the ambulance care national sector organization developed an ambulance care national protocol (ANP). ${ }^{19}$ The ANP is implemented in the national training course for ambulance nurses, and each ambulance nurse receives the ANP during training. In line with the ANP, an ED national protocol (EDNP) for emergency nurses was developed by the Dutch Emergency Nurses Association. ${ }^{20}$ After its publication, the EDNP was disseminated through all emergency departments in the Netherlands. Emergency nurses can consult the EDNP online if they are members of the Dutch Emergency Nurses Association or in a book if present in their emergency department. Because ED training courses are decentrally organized, the EDNP was not implemented in all courses. Both protocols are regularly updated and consist of symptom-orientated algorithms that cover all aspects of prehospital and emergency care. Because both protocols were developed by national professional organizations, they represent the professional nursing standard for ambulance and ED care in the Netherlands.

\section{FRAMEWORK}

To construct the study, Grol's model for effective implementation (Figure 1) was selected as the main framework. ${ }^{1,2}$ The model provides a stepwise approach for improving clinical practice and starts with the identification of research findings, guidelines, or best practices that are intended to be implemented (step 1). Steps 2 and 3 include a description of change targets and an analysis of the target group, current practice, and setting. On the basis of this analysis, implementation strategies can be developed or selected (step 4), followed by the development, execution, evaluation, and adaptation of an implementation plan (steps 5 and 6). Because the ANP and EDNP are already in use in clinical practice, this study focused on the third step to identify factors that influence adherence. Ambulance nurses and emergency nurses (referred to as nurse participants) were included in this study because they represent the intended target group for the ANP and EDNP, respectively. If ambulance nurses and emergency nurses use and adhere to the ANP or EDNP, organizational support is essential and therefore EMS and emergency physicians (referred to as physician participants) were included for context analysis. 


\section{INTERVIEWS}

We used a qualitative design and performed individual, semi-structured interviews that focused on influencing factors related to the individual, the organization, the external context, and the protocols. Interviews were held from the perspectives of nurses and physicians with medical end responsibility in the prehospital and ED setting. Between September 2009 and January 2010, 5 ambulance nurses, 5 emergency nurses, 5 EMS physicians, and 5 emergency physicians were interviewed by the same researcher (R.H.A.E.). Five interviews per discipline were needed to achieve data saturation. Initially, participants were recruited by advertisements on acute care national Websites. Seven participants were selected after their response to the advertisements. Inclusion criteria were that participants (1) were registered as nurses or physicians; (2) could read, write, and speak Dutch; and (c) had a current EMS or ED appointment. Snowball sampling ${ }^{21}$ was used to complete the sample when included participants recommended nurses and physicians. Interviews were audiotaped and were held on a location chosen by the participants, including emergency departments $(n=9)$, EMS offices $(n=8)$, residences $(n=2)$, and the office of the researcher $(n=1)$. Of the 20 participants, 7 were women. The ambulance participants worked in 9 different EMSs, and the ED participants worked in 10 different emergency departments from teaching and university hospitals. The topic list used to structure the interviews contained explorative opening questions ("What can you tell me about factors influencing adherence related to...?") related to the individual, the organization, the external context, and protocol characteristics. $^{2}$ The interviewer made sure that every topic was discussed, but no pre-structured questions besides the explorative opening questions were formulated.

\section{DATA ANALYSIS}

Content analysis ${ }^{21}$ was used to identify influencing factors. Therefore audiotaped interviews were transcribed verbatim and segmented in manageable pieces, which contained 1 or 2 related topics. The second step was coding the text in the segments using open and in vivo codes. To assess inter-rater reliability, a random sample of the segments $(20 \%)$ was drawn, which then were coded and discussed by 2 researchers (R.H.A.E. and D.M.J.S.) until consensus was established. Finally, 2 researchers (R.H.A.E. and D.M.J.S.) assigned the codes into categories of influencing factors until consensus was reached. Whenever possible, identified influencing factors were marked as barriers or facilitators and illustrative quotes were added to the results.
To increase dependability and credibility, data triangulation, investigator triangulation, peer reviews, and member checks were applied. ${ }^{21}$ To ensure data triangulation, data were collected from different individuals from different organizations. Investigator triangulation was accomplished by involving 2 different researchers in the data analysis phase. Reanalyses of raw data were made by a peer researcher (D.M.J.S.). Finally, identified categories of influencing factors were sent to the participants for a member check and supplementary comments. We used the Kwalitan program (version 5.0; Kwalitan, Malden, the Netherlands) for qualitative analysis. During and after data collection and analysis, data were stored on a "stand-alone" computer (with periodic backups stored in a safe), to which only the researcher (R.H.A.E.) and the supervisors (L.C.M.V. and J.A.J.M.) had access.

\section{ETHICAL CONSIDERATIONS}

The recommendations of the Netherlands' Central Committee on Research Involving Human Subjects were executed, following the step-by-step plan research committee review (www.ccmo-online.nl/main.asp?pid=1\&taal=1). For this study, ethical approval of a certified health care ethics committee was not needed, because-by Dutch law -this is not necessary when patients are not exposed to experimental care or treatment, when data collection does not occur at the patient level, when participants are not asked for medical or highly personal information, and when data collection is not burdensome. Although no ethical approval was needed, written informed consent was obtained from every participant before the start of each interview.

\section{Results}

Figure 2 gives an overview of identified influencing factors for the ambulance and ED settings. Results for influencing factors were categorized into individual, organizational, and external factors and protocol characteristics. Because nurse participants were the intended target group, they could mention "individual factors." Opinions of the physician participants were considered as organizational factors.

\section{INFLUENCING FACTORS-AMBULANCE}

\section{Individual factors}

All nurse participants reported how clinical experience facilitates their deviation from the ANP and thereby using the protocol as a guideline: "To me, the protocol is a guideline." These nurse participants believed that a 


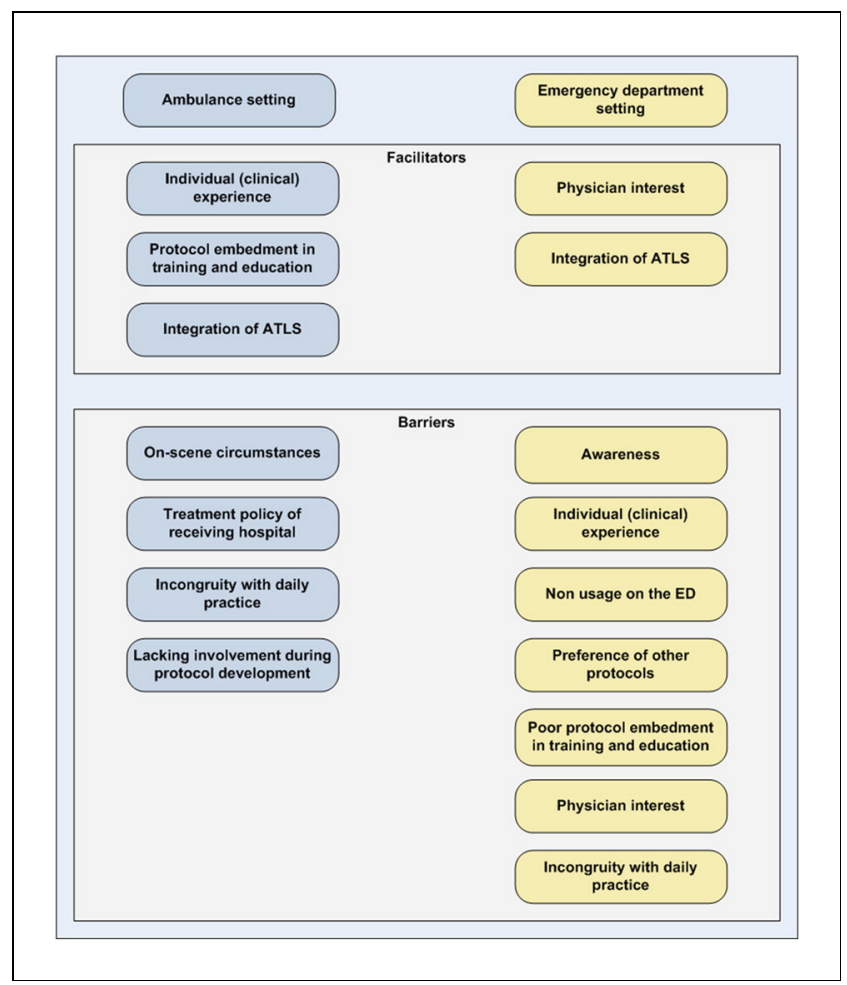

FIGURE 2

Overview of influencing factors.

deviation based on (clinical) experience leads to justified professional care. On the other hand, most nurses used the ANP as an instrument to justify their treatment and feel protected from prosecution when they strictly adhere: "Legally, you are protected when you adhere to the protocol." The ANP is also used as argumentation when decisions were questioned by other professionals, like ED staff or general practitioners.

\section{Organizational factors}

Most physician participants reported that protocol deviations based on ambulance nurses' (clinical) experience are justified and that an ambulance nurse has to be capable of deciding when to deviate: "I expect from the ambulance nurses that, if necessary, they can decide to deviate from the protocol." To monitor protocol adherence, all physician participants pointed out that they check patient records. Nurse participants perceived this check by the physician as disciplinary action: "If they check patient records, the ANP is used against you because you used your common sense." A barrier mentioned by most of the nurse participants was the feeling that they have too little input during the development of the ANP. Regarding this development, nurse participants felt medical dominance by the EMS physician and were afraid that this would lead to a gap between the ANP and daily practice, especially when local adjustments to the protocols are made by the EMS physicians: "Physicians who don't have close contact with daily practice decide what I can and can't do." All nurse participants mentioned that the embedment of the ANP in education and training is a facilitator for adherence: "The national training and protocol belong together." However, some nurse participants felt a need for structural feedback in their organization to discuss protocol use.

\section{External factors}

On-scene circumstances (eg, bystander expectations, aggression, and environmental factors) influenced protocol adherence, especially when the ambulance nurses' own safety was at stake. The treatment policy of the patients' receiving emergency department was also mentioned as a barrier for ambulance nurses' adherence to the ANP: "In case of a high energetic trauma, you go to [named hospital] with the patient. It's a trauma center. Then I think, let's adhere completely to be sure."

\section{Protocol characteristics}

Most nurse and physician participants believed that the ANP is sometimes incongruent with daily practice. They felt that medical treatment according to the ANP is not always appropriate and sufficient and that some medications are not present or do not correspond with other disciplines: "Then you look and you start, but you keep thinking it isn't right, the dosage prescribed isn't present on the ambulance so I can't give it" and "So, apparently, ambulance nurses have to little medication options in their protocol to cope with primary hyperventilation; they get blocked in their protocol." The integration of the advanced trauma life support (ATLS) approach in the ANP was perceived as an essential component that offers assistance for systematic, clinical practice and was perceived as a facilitator for adherence: "Working with the ATLS is an essential condition for functioning as ambulance nurse."

\section{INFLUENCING FACTORS-EMERGENCY DEPARTMENT}

\section{Individual factors}

A barrier for adherence felt by all nurse participants was that awareness of the EDNP is lacking. Nurse participants who were aware of the EDNP out of personal interest stated that most colleagues were not aware of the EDNP. For example, the interviewer asked, "If I understand 
correctly, your colleagues aren't aware of the existence of the EDNP?" The respondent answered, "No, I'm practically sure; maybe a few are." Instead of working with the EDNP, most nurse participants preferred locally developed hospital protocols. Another barrier for protocol adherence in general was when most of the nurse participants stated that they preferably work on the basis of (clinical) experience rather than on the basis of protocols: "Most of my colleagues prefer working on the basis of their experiences and won't look at any protocol."

\section{Organizational factors}

In addition to the awareness factor mentioned by the nurse participants, most of the physician participants also identified the awareness factor as a barrier, relating that they did not know the EDNP existed and had to review it before the interview: "To be honest, I wasn't aware of the EDNP before the interview." Another barrier mentioned was that none of the emergency departments where the nurse participants worked used the EDNP as official ED protocol: "I personally happened to have the EDNP. But it isn't used in this emergency department." Most nurse participants also stated that the EDNP is not embedded, used, or mentioned in training and education: "School never mentioned that there was such thing as a national protocol." Some physician participants mentioned that in the emergency department, an emergency physician is present, and more time exists to administer treatment compared with prehospital care: "Ambulance nurses work solo and have to make decisions on their own. At the emergency department, there is always a physician present who makes treatment policy, with or without protocol."

\section{External factors}

Some nurse participants felt that emergency physicians would be less interested in the EDNP: "When a book states 'nurse' on the cover, they won't look at it." However, all physician participants stated that they were interested in the EDNP so they could integrate nursing and medical practice, which leads to uniformity and absence of equivocality: "It's important to know the content of the protocols colleagues work with" and "It is important for nurses to have a national protocol."

\section{Protocol characteristics}

All nurse participants considered the content of the EDNP as too general and not congruent with $\mathrm{ED}$ practice and therefore a barrier for adherence: "Main disadvantage of the protocol is that it's too general. It isn't specific enough."
The integration of the ATLS approach was considered positive and as leading to a better connection between the EDNP and ANP and, thereby, as a facilitator for adherence.

\section{Discussion}

This qualitative study provided in-depth understanding of factors influencing ambulance and emergency nurses' adherence to ambulance and ED protocols. We identified individual (clinical) experience, awareness, preference of other local protocols, physician interest, protocol embedment in training and education, involvement during protocol development, integration of ATLS, interest of emergency physicians, local circumstances, treatment policy of the receiving hospital, and being in accordance with daily practice as influencing factors. Understanding these factors is important when one is developing and selecting strategies to improve adherence. ${ }^{18}$

Individual clinical experience was found as an influencing factor for adherence to ambulance and ED protocols. Emergency nurses preferred experience over the EDNP and locally developed hospital protocols, so this may be a barrier for emergency nurses to adhere to their professional standard. Emergency nurses preferring their own routines and habits were described earlier by Ebben et $\mathrm{al}^{22}$ In contrast, ambulance nurses felt that their clinical experience was a facilitator for protocol adherence. Literature shows that during emergency situations, nurses use their experiences in the decision-making process by comparing previously experienced situations with the current situation. ${ }^{23,24}$ In this process the similarity of the current patient to a group of patients in the past (representativeness) plays an important role. Because emergency nurses see more patients during a shift compared with ambulance nurses, it is possible that emergency nurses have a larger "reference group" to which to compare the current patient, which may lead to the preference of their own routines and habits. Furthermore, they also have the option to rely on other colleagues and can use locally developed hospital protocols instead of the EDNP.

Another explanation for the difference between ambulance and emergency nurses may be differences in training and education. Ambulance nurses are trained with the ANP and learn to manage it in the context of their individual experience. In contrast, emergency nurses are not trained in their protocol because the EDNP is not always included in their training and education. Therefore it is possible that emergency nurses consider individual experience and protocols as two separate entities whereas ambulance nurses integrate the protocol and individual experience. The 
perception of protocols as authoritative rules of practice that threaten professional autonomy and judgment has been earlier described by Swinkels et al. ${ }^{25}$ Because evidence-based practice acknowledges the integration of individual clinical expertise with external evidence, ${ }^{1}$ using individual experience can sometimes be justified. However, protocol-based care can also be viewed as a mechanism that expands and extends nursing roles and thereby increases professional autonomy. $^{26}$

From the individual as well as the organizational perspective, both emergency nurses and emergency physicians reported poor awareness of the EDNP. In this study the lack of awareness may have led to nonuse of the EDNP and poor embedment in training and education. In a previous study we already identified awareness as a barrier for adherence, ${ }^{22}$ even though awareness seems to be a crucial first step to improve implementation and adherence. In both ambulance and ED settings, it appears that physicians support the protocols and, specific to the ED setting, the physicians are willing to integrate nursing and medical practice. This positive attitude reflects the opportunity to develop integrated guidelines and protocols for nurses and physicians.

Looking at protocol characteristics, for both protocols, the integration of the ATLS approach was described as being a facilitator or even as an essential condition for functioning as an ambulance or emergency nurse. This positive attitude toward ATLS is also shown in a study with physician ATLS trainees. ${ }^{27}$ The incongruity of the ANP with daily practice reported by the ambulance nurses seems consistent with their feeling of too little input during development, which may provide a considerable barrier for adherence. Mrayyan 28 argues that nursing involvement during protocol development can enhance autonomous decision making by nurses, although nursing involvement during guideline and protocol development seems not to be the general rule.

${ }^{29}$ Our results indicate that involvement of intended target users during development of protocols may positively influence adherence and implementation.

\section{LIMITATIONS}

This qualitative study possibly suffered from selection bias in the process of recruiting participants: 7 participants selected themselves by responding to advertisements. These participants may be extremely negative or positive about protocols. However, because influencing factors were our focus rather than the level of adherence, this is not necessarily a problem. This selection bias may have seeped into sampling bias by using snowball sampling to recruit participants. Furthermore, no emergency nurses who were unaware of the EDNP were included. Another limitation considering the emergency nurses was their difficulty separating their experiences with the national protocol from the self-developed hospital protocols, although the self-developed protocols may have been based on the national protocol without their knowing it. Adherence to protocols is important to reduce the incidence of errors occurring through omission of treatment and to ensure that a maximal number of patients receive the benefit of appropriate treatment. ${ }^{10}$ This implies that protocols need to be evidence based and have a positive impact on patient outcomes. In reality, however, not every protocol is evidence based or has a clear relationship with patient outcomes, and therefore deviations might be necessary. Therefore professionals should always use their professional judgment when applying a protocol. Deviations from protocols should be accompanied by strong arguments. However, other reasons, such as standardization and integration of care, can also justify the efforts on improving adherence.

\section{Implications for practice, education, and future research}

For ambulance and emergency nurses, our results can serve as a starting point to identify facilitators and barriers for adherence when implementing protocols. Our results may also contribute to emergency care education and training for nurses by providing information for nursing educators on how to use protocols. Specifically, the tension between professional autonomy and protocols should be addressed. For organizations, it is important to involve a delegation of the intended target group when developing protocols, to prevent nurses from having too little input and to make protocols more congruent with daily practice. Because our study is one of the first studies that identified factors influencing adherence, future research should continue with the identification of these factors. Thereby, it is important to gain in-depth understanding as well as to quantify which influencing factors contribute the most to non-adherence.

We identified influencing factors for adherence to guide the selection and development of implementation strategies. The implementation process can be divided into 5 phases: orientation, insight, acceptance, change, and maintenance. ${ }^{2}$ When classifying the identified influencing factors in these phases, it is shown that the factors influencing adherence to the EDNP are most related to the orientation and insight phases. For the ANP, factors especially relate to the acceptance and maintenance phases. According to this classification, the ambulance protocol is ahead in implementation compared with the ED protocol. 
For clinical practice, this implies that dissemination and implementation strategies should be tailored at these phases.

\section{Conclusion}

This study shows that in the Netherlands, ambulance and emergency nurses' adherence to protocols is influenced by factors related to the individual, the organization, external (social/physical), organization, and protocol characteristics. An influencing factor can be a barrier as well as a facilitator depending on the setting. Therefore implementation strategies should be targeted at these categories and tailored to the setting.

An online copy of the ANP is available at http://lw2.easysite.nl/Ambulanceplein_c01/root/AmbulancezorgHandboek/ index2011.htm. An online copy of the EDNP is available at http://www.lpseh.nl/demo/index.htm.

\section{REFERENCES}

1. Van Achterberg T, Schoonhoven L, Grol R. Nursing implementation science: how evidence-based nursing requires evidence-based implementation. J Nurs Scholarsh. 2008;40(4):302-10.

2. Grol R, Wensing M, Eccles M. Improving Patient Care. The Implementation of Change in Clinical PracticeElsevier Science; 2005.

3. Madan AK, Rentz DE, Wahle MJ, Flint LM. Noncompliance of health care workers with universal precautions during trauma resuscitations. South Med J. 2001;94(3):277-80.

4. Scliopou J, Mader TJ, Durkin L, Stevens M. Paramedic compliance with ACLS epinephrine guidelines in out-of-hospital cardiac arrest. Prehosp Emerg Care. 2006;10(3):394-6.

5. Kirves H, Skrifvars MB, Vahakuopus M, Ekstrom K, Martikainen M, Castren M. Adherence to resuscitation guidelines during prehospital care of cardiac arrest patients. Eur J Emerg Med. 2007;14(2):75-81.

6. Ricard-Hibon A, Belpomme V, Chollet C. Compliance with a morphine protocol and effect on pain relief in out-of-hospital patients. J Emerg Med. 2008;34(3):305-10.

7. Larkin GL, Rolniak S, Hyman KB, MacLeod BA, Savage R. Effect of an administrative intervention on rates of screening for domestic violence in an urban emergency department. Am J Public Health. 2000;90:1444-8.

8. Sebastian RJ, Miller K, Langdorf MI, Johnson D. EMS adherence to a prehospital cervical spine clearance protocol. CalJ Emerg Med. 2001;2(4):44-6.

9. Zegre-Hemsey J, Sommargren CE, Drew BJ. Initial ECG acquisition within 10 minutes of arrival at the emergency department in persons with chest pain: time and gender differences. J Emerg Nurs. 2011;37(1):109-12.

10. Figgis $\mathrm{K}$, Slevin $\mathrm{O}$, Cunningham JB. Investigation of paramedics' compliance with clinical practice guidelines for the management of chest pain. Emerg Med J. 2010;27(2):151-5.

11. Charpentier S, Sagnes-Raffy C, Cournot M. Determinants and prognostic impact of compliance with guidelines in reperfusion therapy for ST-segment elevation myocardial infarction: results from the ESTIM Midi-Pyrenees Area. Arch Cardiovasc Dis. 2009;102(5):387-96.
12. Kelly AM, Powell C, Kerr D. Snapshot of acute asthma: treatment and outcome of patients with acute asthma treated in Australian emergency departments. Intern Med J. 2003;33(9-10):406-13.

13. Musacchio NS, Gehani S, Garofalo R. Emergency department management of adolescents with urinary complaints: missed opportunities. J Adolesc Health. 2009;44(1):81-3.

14. Pham JC, Kelen GD, Pronovost PJ. National study on the quality of emergency department care in the treatment of acute myocardial infarction and pneumonia. Acad Emerg Med. 2007;14(10):856-63.

15. Lee PL, Luo JP, Shieh WM, Nien CT, Yang PC, Kuo SH. Hospital-based management of acute asthmatic exacerbation: an assessment of physicians' behavior in Taiwan. J Asthma. 2001;38(7):575-83

16. Roy PM, Meyer G, Vielle B. Appropriateness of diagnostic management and outcomes of suspected pulmonary embolism. Ann Intern Med. 2006;144(3):157-64.

17. Ferguson CC, Roosevelt G, Bajaj L. Practice patterns of pediatric emergency medicine physicians caring for young febrile infants. Clin Pediatr (Phila). 2010;49(4):350-4.

18. Berben SA, Meijs TH, van Grunsven PM, Schoonhoven L, van Achterberg T. Facilitators and barriers in pain management for trauma patients in the chain of emergency care. Injury. 2012;43(9):1397-402.

19. Sector Organisation Ambulance Care. Ambulance Care National Protocol (ANP) Version 7.2. Zwolle: Stichting, Landelijke Ambulance en Meldkamer Protocollen; 2011.

20. Dutch Emergency Nurses Association. National Protocol Emergency Department (LPSEH). Zwolle: Stichting Landelijke Ambulance en Meldkamer Protocollen and Nederlandse Vereniging Spoedeisende Hulp Verpleegkundigen; 2006.

21. Holloway I, Wheeler S. Qualitative Research in Nursing. Oxford, England: Blackwell Science Ltd; 2004.

22. Ebben RH, Vloet LC, de Groot JM, Van Achterberg T. Factors influencing adherence to an emergency department national protocol. Eur J Emerg Med. 2012;19(1):53-6.

23. Cioffi J. A study of the use of past experiences in clinical decision making in emergency situations. Int J Nurs Stud. 2001;38(5):591-9.

24. Cioffi J. Decision making by emergency nurses in triage assessments. Accid Emerg Nurs. 1998;6(4):184-91.

25. Swinkels A, Albarran JW, Means RI, Mitchell T, Stewart MC Evidence-based practice in health and social care: where are we now? J Interprof Care. 2002;16(4):335-47.

26. Rycroft-Malone J, Fontenla M, Bick D, Seers K. Protocol-based care: impact on roles and service delivery. J Eval Clin Pract. 2008;14(5): 867-73.

27. Campbell B, Heal J, Evans $S$, Marriott $S$. What do trainees think about advanced trauma life support (ATLS)? Ann $R$ Coll Surg Engl. 2000;82(4):263-7.

28. Mrayyan MT. A unit-based protocol to enhance Jordanian nurses' autonomous decision making. J Nurs Manag. 2006;14(5):391-6.

29. Holleman G, Eliens A, Van Vliet M, Van Achterberg T. Promotion of evidence-based practice by professional nursing associations: literature review. J Adv Nurs. 2006;53(6):702-9. 